\title{
FIXED POINTS OF FINITE GROUPS ACTING ON GENERALISED THOMPSON GROUPS
}

\author{
D. H. KOCHLOUKOVA, C. MARTÍNEZ-PÉREZ, AND B. E. A. NUCINKIS
}

\begin{abstract}
We study centralisers of finite order automorphisms of the generalised Thompson groups $F_{n, \infty}$ and conjugacy classes of finite subgroups in finite extensions of $F_{n, \infty}$. In particular we show that centralisers of finite automorphisms in $F_{n, \infty}$ are either of type $\mathrm{FP}_{\infty}$ or not finitely generated.

As an application we deduce the following result about the Bredon type of such finite extensions: any finite extension of $F_{n, \infty}$, where the elements of finite order act on $F_{n, \infty}$ via conjugation with piecewise-linear homeomorphisms, is of type Bredon $\mathrm{F}_{\infty}$. In particular finite extensions of $F=F_{2, \infty}$ are of type Bredon $\mathrm{F}_{\infty}$.
\end{abstract}

\section{INTRODUCTION}

In the 1960s R. Thompson defined the group $F$, which has a realisation as a subgroup of the group of increasing PL (piece-wise linear) homeomorphisms of the unit interval. For a fixed natural number $n$ the group $F$ has a generalization $F_{n}$ given by the infinite presentation

$$
\left.\left\langle x_{0}, x_{1}, \ldots, x_{i}, \ldots\right| x_{i}^{x_{j}}=x_{i+n-1} \text { for } 1 \leq i, 0 \leq j<i\right\rangle .
$$

Furthermore the group $F_{n}$ has a realization, denoted $F_{n, \infty}$, as a subgroup of the group of increasing PL homeomorphisms of the real line $\mathbb{R}$, which we consider in this paper, see [7, Section 2].

The R. Thompson group $F$ satisfies strong cohomological finiteness conditions: it is of homological type $\mathrm{FP}_{\infty}$, is finitely presented [8] but has infinite cohomological dimension. The same properties hold for the generalised R. Thompson groups of [9] and some further generalizations of $F$ as in [21].

The homological property $\mathrm{FP}_{\infty}$ has a homotopical counterpart. The coresponding homotopical condition $\mathrm{F}_{\infty}$ requires that the group admits a finite type model for $E G$, the universal cover of the Eilenberg-Mac Lane space. A group of type $\mathrm{FP}_{\infty}$ is not necessary of type $\mathrm{F}_{\infty}$ [1] and in general a group $G$ is of type $\mathrm{F}_{\infty}$ if and only if it is finitely presented and of type $\mathrm{FP}_{\infty}$. Though the group $F_{n, \infty}$ admits a finite type model for $E G$, such a model is never finite dimensional as $F_{n, \infty}$ has infinite cohomological dimension.

In this paper we consider Bredon cohomological finiteness conditions of finite extensions of generalised Thomspon groups. A Bredon cohomological

Date: January 26, 2010.

2000 Mathematics Subject Classification. 20J05.

The first author is partially supported by "bolsa de produtividade em pesquisa, CNPq". The second author is partially supported by Gobierno de Aragon and MTM2007-68010C03-01. This work was also partially supported by Royal Society International Travel Grant TG08182 and LMS Scheme 4 Grant 4814. 
finiteness condition is a finiteness condition satisfied by groups admitting a cocompact model for $E G$, the classifying space for proper actions. A $G$ CW-complex $X$ is a model for $E G$, if $X^{H}$ is contractible whenever $H \leq G$ is finite and $X^{H}=\varnothing$ otherwise. By definition a group $G$ is of type Bredon $\mathrm{F}_{\infty}$ if it admits a finite type model for $\underline{E}$. For background on Bredon type $\mathrm{F}_{\infty}$ the reader is referred to $[13,19]$. Obviously if the group $G$ is torsion-free, as are the generalised Thompson groups considered here, the Bredon type $\mathrm{F}_{\infty}$ is equivalent to the ordinary homotopical type $\mathrm{F}_{\infty}$.

In [13], W. Lück gave an algebraic criterion for an arbitrary group to admit a finite type model for $\underline{E} G$. In particular, the following two conditions are equivalent:

(i) $G$ is of type Bredon $\mathrm{F}_{\infty}$

(ii) $G$ has finitely many conjugacy classes of finite subgroups and centralisers $C_{G}(H)$ of finite subgroups $H$ of $G$ are of type $\mathrm{F}_{\infty}$.

Although the ordinary finiteness conditions $\mathrm{FP}_{\infty}$ and $\mathrm{F}_{\infty}$ are preserved under finite extensions, it was shown in [12] that generally this does not hold in the Bredon context. In particular, there are examples of finite extensions of groups of type F, i.e. of groups admitting a finite Eilenberg-Mac Lane space, for which either of the above conditions fails. For large classes of groups, finite extensions of groups of type Bredon $\mathrm{F}_{\infty}$ are again of type Bredon $\mathrm{F}_{\infty}$. Examples include hyperbolic groups [18] and soluble groups $[16,11]$.

In this paper we study finite extensions of $F_{n, \infty}$. Lück's result implies that in order to study the Bredon type of finite extensions of $F_{n, \infty}$, we first have to study the group of fixed points of finite order automorphisms of $F_{n, \infty}$. Our first main result points exactly in this direction.

Theorem A. Let $F_{n, \infty}$ be a generalised Thompson group and $\varphi$ be an automorphism of finite order of $F_{n, \infty}$. Then

(1) There is a decreasing homeomorphism $h$ of the real line such that $\varphi(f)=h^{-1} f h, h^{2}=i d$ and there is a unique real number $t_{0}$ such that $\left(t_{0}\right) h=t_{0}$;

(2) The group of the fixed points of $\varphi$ is of type $\mathrm{F}_{\infty}$ if and only if there is an $f \in F_{n, \infty}$ such that $\left(t_{0}\right) f=t_{0}$ and the right-hand slope of $f$ at $t_{0}$ is not 1 .

(3) If $h$ is $P L$ then the group of the fixed points of $\varphi$ is of type $\mathrm{F}_{\infty}$. In particular, if $n=2$ the group of the fixed points of $\varphi$ is of type $\mathrm{F}_{\infty}$.

Part (2) of Theorem A is proved in Sections 4 and 5 and the proof is completed in Section 8. The last line of part (3) of Theorem A follows directly from the fact that the structure of $\operatorname{Aut}(F)$ is well understood, see [6]. In particular, any automorphism of $F$ is given by conjugation with a PL homeomorphism $h$. The case $n \geq 3$ is much more complicated: there are automorphisms of $F_{n, \infty}$ given by conjugation with homeomorphisms of the real line $h$ which are not PL [7]. Such automorphisms are called exotic. In the final Section 10 we exhibit infinitely many exotic automorphisms of finite order for any $n \geq 3$, making substantial use of a construction from [7].

Note that the proof of Lemma 4.11 relies on a Bieri-Strebel-Neumann result about the $\Sigma^{1}$-invariant of finitely generated subgroups of groups of PL 
homeomorphisms of a closed interval [4]. This together with Lemma 4.12 is one crucial ingredient of the proof of Theorem A. In the preliminaries section we include the necessary background for $\Sigma$-theory required later on. It is interesting to note that methods from $\Sigma$-theory were also involved when finiteness conditions of centralisers of finite subgroups of soluble groups where considered in [16].

The following proposition can be deduced as a corollary of Theorem A, but it has an independent short proof, see Corollary 5.2. Note that we do not know of an example where the group of fixed points of $\varphi$ is infinitely generated.

Proposition B. Let $F_{n, \infty}$ be a generalised Thompson group and let $\varphi$ be an automorphism of finite order of $F_{n, \infty}$. Then the group of fixed points of $\varphi$ is either infinitely generated or of type $\mathrm{F}_{\infty}$.

The proof of Proposition B together with Theorem A imply that in the second part of Theorem A the condition $f \in F_{n, \infty}$ can be substituted with $f \in C_{F_{n, \infty}}(\varphi)$, see Corollary 5.3.

The next step in our programme on Bredon finiteness properties is to study the conjugacy classes of finite subgroups in a finite extension of $F_{n, \infty}$. By the first part of Theorem A we obtain that finite order non-trivial automorphisms of $F_{n, \infty}$ have order 2. This motivates the study of conjugacy classes of finite subgroups of split extensions of $F_{n, \infty}$ by the cyclic group of order 2. The reader is referred to Section 7 and Theorem 7.3 in particular. Combining Theorem 7.3 with Theorem A and Lück's criterion, we obtain the main result of this paper :

Theorem C. Any finite extension $G$ of $F_{n, \infty}$ where the elements of finite order act on $F_{n, \infty}$ via conjugation with $P L$ homeomorphisms is Bredon $\mathrm{F}_{\infty}$.

We actually prove a slightly stronger version of Theorem C. In Lemma 4.1 we show that any non-identity finite order automorphism of $F_{n, \infty}$ is given by conjugation with a homeomorphism $h$ of the real line fixing a unique $t_{0} \in \mathbb{R}$. The proof of Theorem $\mathrm{C}$ only requires that $t_{0} \in \frac{1}{2} \mathbb{Z}\left[\frac{1}{n}\right]$ and this is satisfied for piecewise linear $h$. As a corollary of Theorem $\mathrm{C}$ we obtain the following result.

Corollary D. Any finite extension of $F$ is Bredon $\mathrm{F}_{\infty}$.

The proofs of Theorem C and Corollary D are completed in Section 9.

\section{Preliminaries on the generalised R. Thompson groups}

Adopting the notation of [7], we write $F_{n, \infty}$ for the group of PL increasing homeomorphisms $f$ of $\mathbb{R}$ acting on the right on the real line such that the set $X_{f}$ of break points of $f$ is a discrete subset of $\mathbb{Z}\left[\frac{1}{n}\right], f\left(X_{f}\right) \subseteq \mathbb{Z}\left[\frac{1}{n}\right]$ and slopes are integral powers of $n$. Furhtermore, there are integers $i$ and $j$ (depending on $f$ ) with

$$
(x) f=\left\{\begin{array}{l}
x+i(n-1) \text { for } x>M, \\
x+j(n-1) \text { for } x<-M
\end{array}\right.
$$

for sufficiently large $M$ (depending on $f$ again). For $t \in \mathbb{Z}$ we define $F_{n, t}$ to be the subgroup of $F_{n, \infty}$ of all elements which are the identity map on the 
interval $(-\infty, t]$. By [7, Lemma 2.1.2] $F_{n, 0}$ is isomorphic to the generalised Thompson group $F_{n}$. Furthermore, by [7, Lemma 2.1.6] $F_{n, \infty} \simeq F_{n, t}$ for every $t \in \mathbb{Z}$. Similarly, for $t \in \mathbb{R}$ we define

$$
F_{n, t}=\left\{f \in F_{n, \infty} \mid \text { the restriction of } f \text { on }(-\infty, t] \text { is the identity map }\right\}
$$

Lemma 2.1. If $t \in \mathbb{Z}\left[\frac{1}{n}\right]$ then $F_{n, t} \simeq F_{n, 0}$. In particular $F_{n, t}$ is of type $\mathrm{F}_{\infty}$.

Proof. The map $\theta: F_{n, t} \rightarrow F_{n, 0}$ defined by $\theta(f)=g f g^{-1}$, where $(x) g=x+t$, is an isomorphism.

\section{Preliminaries on Sigma theory}

In this section we collect facts about the homological $\Sigma$ invariants, which we shall use in Section 4. Note that there is also a homotopical version, but we will not make any use of it here. Let $G$ be a finitely generated group and $\chi: G \rightarrow \mathbb{R}$ be a non-zero character. We define the monoid

$$
G_{\chi}=\{g \in G \mid \chi(g) \geq 0\}
$$

and the character sphere

$$
S(G)=\operatorname{Hom}(G, \mathbb{R}) \backslash\{0\} / \sim,
$$

where $\sim$ is an equivalence relation on $\operatorname{Hom}(G, \mathbb{R}) \backslash\{0\}$ defined in the following way : $\chi_{1} \sim \chi_{2}$ if and only if there is a positive real number $r$ such that $r \chi_{1}=\chi_{2}$. By definition, see [5], for the trivial right $\mathbb{Z} G$-module $\mathbb{Z}$

$$
\Sigma^{m}(G, \mathbb{Z})=\left\{[\chi] \in S(G) \mid \mathbb{Z} \text { is } \mathrm{FP}_{m} \text { over } \mathbb{Z} G_{\chi}\right\},
$$

where $[\chi]$ denotes the equivalence class of $\chi$ and $m \geq 0$ or $m=\infty$.

3.1. Meinert's results. The following is a result due to H. Meinert, but the proof was published in [20, Lemma 9.1].

Lemma 3.1. Let $G_{1}$ and $G_{2}$ be groups of type $\mathrm{FP}_{d+1}$, and let $i$ and $j$ be non-negative integers such that $i+j \leq d$. Then

$$
\pi_{1}^{*}\left(\Sigma^{i}\left(G_{1}, \mathbb{Z}\right)\right)+\pi_{2}^{*}\left(\Sigma^{j}\left(G_{2}, \mathbb{Z}\right)\right) \subseteq \Sigma^{i+j+1}\left(G_{1} \times G_{2}, \mathbb{Z}\right)
$$

where $\pi_{i}^{*}: S\left(G_{i}\right) \rightarrow S\left(G_{1} \times G_{2}\right)$ is the map induced by the canonical projection $\pi_{i}: G_{1} \times G_{2} \rightarrow G_{i}$.

Corollary 3.2. Let $G_{1}$ and $G_{2}$ be groups of type $\mathrm{FP}_{\infty}$, and for $i \in\{1,2\}$ let $\chi_{i} \in \operatorname{Hom}\left(G_{i}, \mathbb{R}\right) \backslash\{0\}$. Furthermore suppose that $\left[\chi_{i}\right] \in \Sigma^{\infty}\left(G_{i}, \mathbb{Z}\right)$ for at least one $i \in\{1,2\}$.

Then for $G=G_{1} \times G_{2}$ and $\chi=\left(\chi_{1}, \chi_{2}\right) \in \operatorname{Hom}(G, \mathbb{R}) \backslash\{0\}$ we have that $[\chi] \in \Sigma^{\infty}(G, \mathbb{Z})$.

Proposition 3.3. [14, Prop. 4.2], [15, Thm. 4.3] Let $H$ be a group of type $\mathrm{F}_{m}$ and $\varphi \in \operatorname{End}(H)$. Put $G=\left\langle H, t \mid t^{-1} H t=\varphi(H)\right\rangle$. Suppose $\chi: G \rightarrow \mathbb{R}$ is a real character such that $\chi(H)=0$ and $\chi(t)=1$. Then $-[\chi] \in \Sigma^{m}(G, \mathbb{Z})$. 
3.2. $\Sigma^{1}$ for PL groups. Let $G$ be a finitely generated group of PL orientation preserving homeomorphisms of an interval $I=\left[y_{1}, y_{2}\right] \subset \mathbb{R}$ acting on the right. Let $\chi_{1}, \chi_{2}: G \rightarrow \mathbb{R}$ be the characters given by $\chi_{i}(f)=\log _{m}\left(y_{i}\right) f^{\prime}$, where $m>1$ is a fixed number.

Theorem 3.4. [4, Thm. 8.1] Assume that $G$ does not fix any point in the open interval $\left(y_{1}, y_{2}\right)$. Further assume that $\chi_{1}\left(\operatorname{Ker}\left(\chi_{2}\right)\right)=\operatorname{Im}\left(\chi_{1}\right)$ and $\chi_{2}\left(\operatorname{Ker}\left(\chi_{1}\right)\right)=\operatorname{Im}\left(\chi_{2}\right)$. Then

$$
\Sigma^{1}(G, \mathbb{Z})=S(G) \backslash\left\{\left[\chi_{1}\right],\left[\chi_{2}\right]\right\} .
$$

Except of the case $G=F=F_{2, \infty}$ [3], there are, untill now, no known results about the higher dimensional $\Sigma$-invariants of groups $G$ of PL orientation preserving homeomorphisms of closed intervals.

3.3. Direct products formula in dimension 1. In small dimensions, the homological $\Sigma$-invariants of direct product of groups can easily be calculated via the $\Sigma$-invariants of the direct factors. There is a version of the direct product formula for $\Sigma$-invariants with coefficients in a field without restriction of the dimension [2]. But we will not make use of this result.

For a group $G$ denote

$$
\Sigma^{1}(G, \mathbb{Z})^{c}=S(G) \backslash \Sigma^{1}(G, \mathbb{Z}) .
$$

The following proposition is the direct product formula in dimension 1 .

\section{Proposition 3.5.}

$$
\begin{aligned}
& \Sigma^{1}\left(G_{1} \times G_{2}, \mathbb{Z}\right)^{c}= \\
& \quad\left\{\left[\left(\chi_{1}, \chi_{2}\right)\right] \mid \text { one of } \chi_{1}, \chi_{2} \text { is zero and for } \chi_{i} \neq 0,\left[\chi_{i}\right] \in \Sigma^{1}\left(G_{i}, \mathbb{Z}\right)^{c}\right\} .
\end{aligned}
$$

Proof. We apply Lemma 3.1 to $i=j=0$ to get

$$
\Sigma^{1}\left(G_{1} \times G_{2}, \mathbb{Z}\right)^{c} \subseteq\left\{\left[\left(\chi_{1}, \chi_{2}\right)\right] \mid \text { one of } \chi_{1}, \chi_{2} \text { is zero }\right\} .
$$

The result follows easily from the definition of $\Sigma^{1}$ taking also into account that the property $\mathrm{FP}_{1}$ behaves well for quotients.

\section{Fixed Points of AUtomorphisms of Finite order}

Lemma 4.1. Every non-identity finite order automorphism $\varphi$ of $F_{n, \infty}$ has order 2. Furthermore $\varphi(f)=h^{-1}$ fh for a unique homeomorphism $h$ of the real line $\mathbb{R}, h^{2}$ is the identity map and $h$ is a decreasing function, so there is an unique $t_{0} \in \mathbb{R}$ such that $\left(t_{0}\right) h=t_{0}$.

Proof. By [17] and [7, Lemma 6.1.1, Lemma 6.1.2], for any $\varphi \in \operatorname{Aut}\left(F_{n, \infty}\right)$ there is a unique homeomorphim $h$ of $\mathbb{R}$ such that

$$
\varphi(f)=h^{-1} f h .
$$

In the case when $n=2$ it was shown in [6, Lemma 5.1] that $h$ is piecewise linear but this does not hold for $n \geq 3$ [7, Thm. 6.1.5]. Now assume that $\varphi$ has finite order, say $k$. Then by the uniqueness of the conjugating homeomorphism in (1) we deduce that $h^{k}=i d$.

We remind the reader that, following the notation of [7], all homeomorphisms of the real line act on the right. For the homeomorphism $h$ there are just two possibilities: increasing or decreasing. In the first case 
consider $x_{0}$ such that $x_{0} \neq\left(x_{0}\right) h$. If $x_{0}<\left(x_{0}\right) h$ since $h$ is increasing $x_{0}<\left(x_{0}\right) h<\left(x_{0}\right) h^{2}<\ldots<\left(x_{0}\right) h^{k}$, giving a contradiction. If $x_{0}>\left(x_{0}\right) h$, again using that $h$ is increasing, $x_{0}>\left(x_{0}\right) h>\left(x_{0}\right) h^{2}>\ldots>\left(x_{0}\right) h^{k}$, giving a contradiction as well. Then $h$ is decreasing and so the function $(x) g=(x) h-x$ is decreasing and there is an unique $t_{0} \in \mathbb{R}$ such that $\left(t_{0}\right) g=0$. Finally, observe that $h^{2}$ is increasing and of finite order and therefore $h^{2}=1$.

Using the notation of Lemma 4.1 we set

$$
C_{F_{n, \infty}}(\varphi)=\left\{f \in F_{n, \infty} \mid \varphi(f)=f\right\} .
$$

Lemma 4.2. $C_{F_{n, \infty}}(\varphi)$ fixes the point $t_{0}$.

Proof. If $f \in C_{F_{n, \infty}}(\varphi)$ we have $h f=f h$. So $\left(t_{0}\right) f h=\left(t_{0}\right) h f=\left(t_{0}\right) f$. Since by Lemma $4.1 t_{0}$ is the unique fixed point of $h$, we deduce that $\left(t_{0}\right) f=t_{0}$.

Proposition 4.3. If $t_{0} \in \mathbb{Z}\left[\frac{1}{n}\right]$ then $C_{F_{n, \infty}}(\varphi)$ is of type $\mathrm{F}_{\infty}$.

Proof. We claim that

$$
C_{F_{n, \infty}}(\varphi) \cong F_{n, t_{0}} .
$$

Consider the map $\Theta: C_{F_{n, \infty}}(\varphi) \rightarrow F_{n, t_{0}}$ defined by $\Theta(f)=\tilde{f}$, where $\tilde{f}$ coincides with $f$ on the interval $\left[t_{0}, \infty\right)$ and is the identity on $\left(-\infty, t_{0}\right]$. The condition $f h=h f$ implies that $\Theta$ is injective. Given $f \in F_{n, t_{0}}$, we can find an element $\hat{f} \in F_{n, \infty}$ satisfying $\varphi(\hat{f})=h \hat{f} h=\hat{f}$ and $\Theta(\hat{f})=f$ as follows:

$$
(t) \hat{f}=\left\{\begin{array}{l}
(t) f \text { for } t>t_{0} \\
(t) h f h \text { for } t \leq t_{0} .
\end{array}\right.
$$

Here we have used that $h$ is decreasing of order 2 and is fixing $t_{0}$.

To finish the proof we use that Lemma 2.1 shows that $F_{n, t_{0}}$ is of type $\mathrm{F}_{\infty}$.

In the rest of this section we complete the proof of one of the directions of Theorem A, part (2), see Corollary 4.15. More precisely, we show that if there is some $f \in F_{n, \infty}$ such that $\left(t_{0}\right) f=t_{0}$ and the right-hand slope of $f$ at $t_{0}$ is not 1 , then $C_{F_{n, \infty}}(\varphi)$ is of type $\mathrm{FP}_{\infty}$. By Proposition 4.3 we can assume that

$$
t_{0} \notin \mathbb{Z}\left[\frac{1}{n}\right]
$$

Note that [6, Lemma 5.1] implies that for $n=2$ the homeomorphism $h$ is $\mathrm{PL}$. Together with some technical results proved independently later on, see the first part of Theorem 7.3, this implies that for $n=2$ we have $t_{0} \in \mathbb{Z}\left[\frac{1}{n}\right]$. In what follows we can therefore assume that

$$
n \geq 3 \text {. }
$$

Definition. Let $I=[\alpha, \beta]$ be an interval in $\mathbb{R}$. By some abuse of notation we also include the cases $\alpha=-\infty$ and $\beta=\infty$. We set

$$
F_{n, I}=\left\{f \mid f \text { is the restriction to } I \text { of } \tilde{f} \in F_{n, \infty}, \tilde{f}(\alpha)=\alpha, \tilde{f}(\beta)=\beta\right\} .
$$

In this section we consider the following condition:

There is an element $\hat{f} \in F_{n, \infty}$, such that $\left(t_{0}\right) \hat{f}=t_{0}$ and the slope of $\hat{f}$ at $t_{0}$ is not 1 . 
Moreover by considering either $\hat{f}$ or $\hat{f}^{-1}$, we may assume $\log _{n}\left(\left(t_{0}\right) \hat{f}^{\prime}\right)<0$ and that $\left|\log _{n}\left(\left(t_{0}\right) \hat{f}^{\prime}\right)\right|$ is as small as possible, that is, the slope at $t_{0}$ is smaller than 1 but as close as possible to 1 . Throughout this section we write $\hat{f}$ for this element.

We also consider the characters $\mu_{1}, \mu_{2}: F_{n,\left[t_{0}, \infty\right]} \rightarrow \mathbb{R}$ given by

$$
\begin{aligned}
& \mu_{1}(f)=\log _{n}\left(\left(t_{0}\right) f^{\prime}\right) \quad \text { and } \\
& \mu_{2}(f)=-i,
\end{aligned}
$$

where $(x) f=x+i(n-1)$ for sufficiently large $x$.

Proposition 4.4. Suppose that (2) holds. Then the group $F_{n,\left[t_{0}, \infty\right]}$ is an ascending HNN extension with a base group isomorphic to $F_{n, \infty}$. In particular, $F_{n,\left[t_{0}, \infty\right]}$ is of type $\mathrm{F}_{\infty}$. Similarly $F_{n,\left[-\infty, t_{0}\right]}$ is of type $\mathrm{F}_{\infty}$.

Proof. Define $M$ to be the subgroup of $F_{n,\left[t_{0}, \infty\right]}$ containing those elements with slope 1 at $t_{0}$, i.e., $M=\operatorname{Ker}\left(\mu_{1}\right)$. Set $\widetilde{f}$ to be the restriction of $\hat{f}$ to $\left[t_{0}, \infty\right]$. Note that the fact that $\mu_{1}$ is a discrete character and the choice of $\widetilde{f}$ imply that $F_{n,\left[t_{0}, \infty\right]}=\langle M, \widetilde{f}\rangle$.

Let $r$ be an element of $\mathbb{Z}\left[\frac{1}{n}\right]$ such that $r>t_{0}$. We identify $F_{n, r}$ with its restriction on the interval $\left[t_{0}, \infty\right)$ i.e. we identify $F_{n, r}$ with its image in $F_{n,\left[t_{0}, \infty\right]}$. Thus if $r_{1}>r_{2}>\ldots>r_{i}>\ldots$ is a decreasing sequence of elements of $\mathbb{Z}\left[\frac{1}{n}\right]$ with limit $t_{0}$ we get that $M=\cup_{i} F_{n, r_{i}}$.

Note that for $r$ as in the previous paragraph, with $r$ sufficiently close to $t_{0}$, the restriction of $\widetilde{f}$ on the interval $\left[t_{0}, r\right]$ is linear. Since the slope of $\widetilde{f}$ at $t_{0}$ is smaller that 1 , we have $t_{0}=\left(t_{0}\right) \tilde{f}<(r) \tilde{f}<r$. Hence

$$
F_{n, r}^{\widetilde{f}}=\widetilde{f}^{-1} F_{n, r} \widetilde{f}=F_{n,(r) \widetilde{f}} \supseteq F_{n, r},
$$

and also for $k \in \mathbb{N}$

$$
F_{n, r}^{\widetilde{f}^{k}}=F_{n,(r) \widetilde{f}^{k}} \supseteq F_{n, r}^{\widetilde{f}^{k-1}}=F_{n,(r) \widetilde{f}^{k-1}} \supseteq \cdots
$$

Define $r_{k}=(r) \widetilde{f}^{k}$. Then the sequence $\left\{r_{k}\right\}$ satisfies the assumptions of the previous paragraph. Thus

$$
M=\cup_{k \geq 1} F_{n, r}^{\widetilde{f}^{k}}
$$

and by $(3) F_{n,\left[t_{0}, \infty\right]}$ is an ascending HNN extension with a base group $F_{n, r}$ and stable letter $\widetilde{f}$. By Lemma $2.1 F_{n, r} \cong F_{n, \infty}$ which is of type $\mathrm{F}_{\infty}$.

Lemma 4.5. The group $F_{n,\left[t_{0}, \infty\right]}$ does not fix any element of $\left(t_{0}, \infty\right)$.

Proof. Let $t_{1} \in\left(t_{0}, \infty\right)$ and $r \in \mathbb{Z}\left[\frac{1}{n}\right] \cap\left(t_{0}, t_{1}\right)$. As $F_{n, r}$ does not fix an element of $(r, \infty)$ and $F_{n, r}$ embeds in $F_{n,\left[t_{0}, \infty\right]}$, we deduce that $F_{n,\left[t_{0}, \infty\right]}$ does not fix $t_{1}$.

Lemma 4.6. The characters $\mu_{1}, \mu_{2}: F_{n,\left[t_{0}, \infty\right]} \rightarrow \mathbb{R}$ satisfy $\mu_{1}\left(\operatorname{Ker}\left(\mu_{2}\right)\right)=$ $\operatorname{Im}\left(\mu_{1}\right)$ and $\mu_{2}\left(\operatorname{Ker}\left(\mu_{1}\right)\right)=\operatorname{Im}\left(\mu_{2}\right)$.

Proof. Again, let $\tilde{f}$ be the restriction of $\hat{f}$ to $\left[t_{0}, \infty\right)$. Choose for any $i \in \mathbb{Z}$ an $\hat{f}_{i} \in F_{n, \infty}$ such that $(x) \hat{f}_{i}=x+i(n-1)$ for sufficiently large $x$ and $\left(t_{0}\right) \hat{f}_{i}=t_{0}$. This is possible, as we can choose $\hat{f}_{i}$ to be the identity map on an interval $I_{i}$ containing $t_{0}$ with end points in $\mathbb{Z}\left[\frac{1}{n}\right]$. Denote by $f_{i}$ the 
restriction of $\hat{f}_{i}$ to $\left[t_{0}, \infty\right]$. Then $\mu_{1}\left(f_{1}\right)=0, \mu_{2}\left(f_{1}\right)=-1$, i.e., $f_{1} \in \operatorname{Ker}\left(\mu_{1}\right)$ and $\mu_{2}\left(f_{1}\right)$ generates $\operatorname{Im}\left(\mu_{2}\right)$. Moreover, for any $i \in \mathbb{Z}$

$$
\mu_{1}\left(\widetilde{f} f_{i}\right)=\mu_{1}(\widetilde{f})+\mu_{1}\left(f_{i}\right)=\mu_{1}(\widetilde{f})
$$

and

$$
\mu_{2}\left(\widetilde{f} f_{i}\right)=\mu_{2}(\widetilde{f})+\mu_{2}\left(f_{i}\right)=\mu_{2}(\widetilde{f})-i .
$$

So we may choose some $i$ with $\mu_{2}\left(\widetilde{f} f_{i}\right)=0$, but $\mu_{1}\left(\widetilde{f} f_{i}\right)=\mu_{1}(\widetilde{f})$ is a generator of $\operatorname{Im}\left(\mu_{1}\right)$ by the choice of $\hat{f}$.

Without loss of generality we can assume that $t_{0} \in(0,1)$. Indeed, otherwise we can interchange $h$ with another homeomorphism $g^{-1} h g$, where $(x) g=$ $x+i$ for some $i \in \mathbb{Z}$ to reduce to $t_{0} \in[0,1)$. Since $t_{0} \notin \mathbb{Z}\left[\frac{1}{n}\right]$, we have $t_{0} \neq 0$.

Lemma 4.7. The group $F_{n,\left[t_{0}, \infty\right]}$ has a realization as a subgroup of the group $H$ of piecewise linear transformations of the interval $\left[t_{0}, n-1\right]$.

Furthermore, the characters $\chi_{1}$ and $\chi_{2}$ of $H$ given by $\log _{n}$ of derivatives at $t_{0}$ and $n-1$ correspond to the characters $\mu_{1}, \mu_{2}: F_{n,\left[t_{0}, \infty\right]} \rightarrow \mathbb{R}$ given by $\mu_{1}(f)=\log _{n}\left(\left(t_{0}\right) f^{\prime}\right)$ and $\mu_{2}(f)=-i$ if at infinity $(x) f=x+i(n-1)$.

Proof. By [7, Lemma 2.3.1] there is a PL homeomorphism $\mu$ between $[0, \infty)$ and $[0, n-1)$ inducing, by conjugation, an isomorphism $\mu^{*}$ between $F_{n, 0}$ and a subgroup of $P L([0, n-1])$. Since $t_{0} \notin \mathbb{Z}\left[\frac{1}{n}\right]$, we cannot directly apply the isomorphism $\mu^{*}$ above. Since by definition $\mu$ is the identity on $[0, n-2]$ and $t_{0}<1 \leq n-2$, the restriction of $\mu$ to $\left[t_{0}, \infty\right)$ induces, via conjugation, an isomorphism between $F_{n,\left[t_{0}, \infty\right]}$ and a subgroup of $P L\left(\left[t_{0}, n-1\right]\right)$. The behaviour at $+\infty$ is explained in the second paragraph of the proof of $[7$, Lemma 2.3.1]: if $f \in F_{n,\left[t_{0}, \infty\right]}$ is such that $(x) f=x+(n-1) i$ for sufficiently large $x$ then the image of $f$ in $P L\left(\left[t_{0}, n-1\right]\right)$ has derivative at $n-1$ equal to $n^{-i}$.

Corollary 4.8. If (2) holds then $\Sigma^{1}\left(F_{n,\left[t_{0}, \infty\right]}\right)^{c}=\left\{\left[\mu_{1}\right],\left[\mu_{2}\right]\right\}$.

Proof. Follows directly from Theorem 3.4, Lemma 4.5, Lemma 4.6 and Lemma 4.7.

Let $\nu_{1}, \nu_{2}: F_{n,\left[-\infty, t_{0}\right]} \rightarrow \mathbb{R}$ be characters defined as follows:

$$
\nu_{1}(f)=\log _{n}\left(\left(t_{0}\right) f^{\prime}\right)
$$

and

$$
\nu_{2}(f)=-i \text { where }(x) f=x+i(n-1) \text { for } x<-M
$$

for some positive number $M$ depending on $f$.

The proof of the following result is the same as the proof of the previous corollary.

Corollary 4.9. If (2) holds then $\Sigma^{1}\left(F_{n,\left[-\infty, t_{0}\right]}\right)^{c}=\left\{\left[\nu_{1}\right],\left[\nu_{2}\right]\right\}$.

Set

$$
D=F_{n,\left[-\infty, t_{0}\right]} \times F_{n,\left[t_{0}, \infty\right]}
$$

and consider the characters $\tilde{\mu}_{1}, \tilde{\mu}_{2}: D \rightarrow \mathbb{R}$ that extend $\mu_{1}, \mu_{2}$ and are the zero map on $F_{n,\left[-\infty, t_{0}\right]}$. Similarly define $\tilde{\nu}_{1}, \tilde{\nu}_{2}: D \rightarrow \mathbb{R}$ to extend $\nu_{1}, \nu_{2}$ and are the zero map on $F_{n,\left[t_{0}, \infty\right]}$. Let $\widetilde{\varphi}$ be the automorphism of Homeo $(\mathbb{R})$ sending $f$ to $h^{-1} f h$, i.e. $\widetilde{\varphi}$ extends $\varphi$. 
Lemma 4.10. If (2) holds then $\widetilde{\varphi}$ is an automorphism of $D=F_{n,\left[-\infty, t_{0}\right]} \times$ $F_{n,\left[t_{0}, \infty\right]}$

Proof. Let $G$ be the subgroup of those elements of $D$ that are differenciable at $t_{0}$ i.e. $t_{0}$ is not a break point, so $G$ is the kernel of $\tilde{\mu}_{1}-\tilde{\nu}_{1}$ and so $D / G \simeq \mathbb{Z}$. Observe that

$$
G=\left\{f \in F_{n, \infty} \mid\left(t_{0}\right) f=t_{0}\right\} .
$$

Since $\left(t_{0}\right) h=t_{0}$ the group $G$ is invariant under $\varphi$ i.e. $\widetilde{\varphi}(G)=G$. To complete the proof it suffices to take any element $f \in D \backslash G$ that is a generator of $D / G \simeq \mathbb{Z}$ and show that $\widetilde{\varphi}(f)=h^{-1} f h \in D$. Then $\widetilde{\varphi}(D) \subseteq D$ and as $\widetilde{\varphi}^{2}=i d$ we get that $\widetilde{\varphi}(D)=D$.

Take $f$ that is the identity map on $\left(-\infty, t_{0}\right]$. Then $\widetilde{\varphi}(f)$ is the identity map on $\left[t_{0}, \infty\right)$. Note that $f$ coincides with some $g \in F_{n, \infty}$ on the interval $\left[t_{0}, \infty\right)$. Then $\widetilde{\varphi}(f)$ and $\varphi(g)$ coincide on $\left(-\infty, t_{0}\right]$. Thus $\widetilde{\varphi}(f) \in D$.

Lemma 4.11. If (2) holds then the map $\widetilde{\varphi}$ induces a map $\widetilde{\varphi}^{*}: S(D) \rightarrow S(D)$ that swaps $\left[\tilde{\mu}_{i}\right]$ with $\left[\tilde{\nu}_{i}\right]$ for $i=1,2$.

Proof. By Corollary 4.8, Corollary 4.9 and the direct product formula in dimension 1 , see Proposition 3.5 , we have that $\Sigma^{1}(D, \mathbb{Z})^{c}$ is exactly the set $\left\{\tilde{\mu}_{i}, \tilde{\nu}_{i}\right\}_{i=1,2}$. Any automorphism of $D$ induces a map that permutes the elements of $\Sigma^{1}(D, \mathbb{Z})^{c}$.

Since $\varphi$ is given by conjugation with a decreasing $h$, we get that $\widetilde{\varphi}^{*}$ permutes the characters corresponding to $\pm \infty$ i.e. $\widetilde{\varphi}^{*}\left(\left[\nu_{2}\right]\right)=\left[\mu_{2}\right]$ and $\widetilde{\varphi}^{*}$ permutes the characters corresponding to derivatives (right or left) at $t_{0}$ i.e $\widetilde{\varphi}^{*}\left(\left[\nu_{1}\right]\right)=\left[\mu_{1}\right]$.

Corollary 4.12. If (2) holds then the map $\widetilde{\varphi}$ induces a map

$$
\widetilde{\varphi}^{* *}: \operatorname{Hom}(D, \mathbb{R}) \rightarrow \operatorname{Hom}(D, \mathbb{R})
$$

swapping $\tilde{\mu}_{i}$ with $\tilde{\nu}_{i}$ for $i=1,2$.

Proof. By Lemma 4.11 there are some positive real numbers $r_{1}$ and $r_{2}$ such that $\tilde{\varphi}^{* *}\left(\tilde{\mu}_{1}\right)=r_{1} \tilde{\nu}_{1}$ and $\widetilde{\varphi}^{* *}\left(\tilde{\mu}_{2}\right)=r_{2} \tilde{\nu}_{2}$. Then for every $f \in F_{n,\left[-\infty, t_{0}\right]}$ we have that $\left(t_{0}\right)\left(h^{-1} f h\right)^{\prime}=\widetilde{\varphi}^{* *}\left(\tilde{\mu}_{1}\right)(f)=r_{1} \tilde{\nu}_{1}(f)=r_{1}\left(\left(t_{0}\right) f^{\prime}\right)$ where derivatives mean right or left derivatives. Applying this to the identity map $f$ we deduce that $r_{1}=1$. Similarly $r_{2}=1$.

The following corollary is not needed to establish the main result of this section i.e. Corollary 4.15, but we include it as it follows easily from the results on $\Sigma$-theory developed in [5].

Corollary 4.13. If (2) holds then the group $G$ defined in the proof of Lemma 4.10 is of type $\mathrm{FP}_{\infty}$.

Proof. By Proposition 4.4 and Proposition 3.3

$$
-\left[\mu_{1}\right] \in \Sigma^{\infty}\left(F_{n,\left[t_{0}, \infty\right]}, \mathbb{Z}\right)
$$

and similarly

$$
-\left[\nu_{1}\right] \in \Sigma^{\infty}\left(F_{n,\left[-\infty, t_{0}\right]}, \mathbb{Z}\right) .
$$

Then by Corollary 3.2 the images of both $\tilde{\mu}_{1}-\tilde{\nu}_{1}=\left(-\nu_{1}, \mu_{1}\right) \in \operatorname{Hom}(D, \mathbb{R})$ and $-\tilde{\mu}_{1}+\tilde{\nu}_{1}=\left(\nu_{1},-\mu_{1}\right) \in \operatorname{Hom}(D, \mathbb{R})$ represent elements of $\Sigma^{\infty}(D, \mathbb{Z})$ i.e. $\left[\left(-\nu_{1}, \mu_{1}\right)\right],\left[\left(\nu_{1},-\mu_{1}\right)\right] \in \Sigma^{\infty}(D, \mathbb{Z})$. Then by $\left[5\right.$, Thm. B] $G=\operatorname{Ker}\left(\tilde{\mu}_{1}-\tilde{\nu}_{1}\right)$ is of type $\mathrm{FP}_{\infty}$. 
Theorem 4.14. If (2) holds then $C_{F_{n, \infty}}(\varphi) \simeq F_{n,\left[t_{0}, \infty\right]}$.

Proof. Observe that

$$
C_{F_{n, \infty}}(\varphi)=\left\{f \in F_{n, \infty} \mid \varphi(f)=f\right\}=\left\{f \in F_{n, \infty} \mid h f=f h\right\} .
$$

Consider the morphism

$$
\rho: C_{F_{n, \infty}}(\varphi) \rightarrow F_{n,\left[t_{0}, \infty\right)}
$$

sending $f$ to its restriction on $\left[t_{0}, \infty\right)$. Similarly to the proof of Proposition 4.3 we now show that $\rho$ is bijective. The fact that $\rho$ is injective is obvious. To show that $\rho$ is surjective consider $f_{1} \in F_{n,\left[t_{0}, \infty\right)}$. Then there is a unique function $\tilde{f}: \mathbb{R} \rightarrow \mathbb{R}$ that coincides with $f_{1}$ on $\left[t_{0}, \infty\right)$ and $\tilde{f} h=h \tilde{f}$. In fact $\tilde{f}=\left(f_{2}, f_{1}\right) \in F_{n,\left[-\infty, t_{0}\right]} \times F_{n,\left[t_{0}, \infty\right]}$. By Corollary $4.12 \widetilde{\varphi}$ induces a map swapping the characters $\tilde{\mu}_{1}$ and that $\tilde{\nu}_{1}$. Since $\widetilde{\varphi}\left(f_{1}\right)=f_{2}, t_{0}$ is not a break point of $f$ and so $f \in F_{n, \infty}$. Thus $f \in C_{F_{n, \infty}}(\varphi)$ and $\rho(f)=f_{1}$ i.e. $\rho$ is surjective.

Theorem 4.14 together with Proposition 4.4 yields the desired direction of Theorem A, part (2).

Corollary 4.15. If (2) holds then $C_{F_{n, \infty}}(\varphi)$ is of type $\mathrm{F}_{\infty}$.

\section{Proof of Proposition B}

We begin by completing the proof of part (2) of Theorem A. This follows from combining the results of the previous section with the following lemma.

Lemma 5.1. Suppose that $C_{F_{n, \infty}}(\varphi)$ is finitely generated. Then there exists an element $f \in C_{F_{n, \infty}}(\varphi)$ with slope at $t_{0}$ not equal to one.

Proof. The case when $t_{0} \in \mathbb{Z}\left[\frac{1}{n}\right]$ is obvious, so we can assume $t_{0} \notin \mathbb{Z}\left[\frac{1}{n}\right]$. Suppose that for every $f \in C_{F_{n, \infty}}(\varphi)$ the slope at $t_{0}$ is 1 . Recall that by Lemma $4.2\left(t_{0}\right) f=t_{0}$. As $C_{F_{n, \infty}}(\varphi)$ is finitely generated there is a small closed interval $J$ containing $t_{0}$ such that the restriction of any element of $C_{F_{n, \infty}}(\varphi)$ to $J$ is the identity. In fact, $J$ is the intersection of the intervals defined for the finitely many generators $f$ of $C_{F_{n, \infty}}(\varphi)$. Thus $J$ is a closed interval, which is not a point.

Let $r_{0}$ be an element of $\mathbb{Z}\left[\frac{1}{n}\right]$ such that $r_{0}>t_{0}$ and $r_{0}$ is in the interior of the interval $J$. Set $f_{0} \in F_{n, r_{0}}$ such that the right-hand derivative at $r_{0}$ is not 1 and denote by $f_{1}$ the restriction of $f_{0}$ on $\left[t_{0}, \infty\right)$. Thus $f_{1} \in F_{n,\left[t_{0}, \infty\right]}$ and $f_{2}=h^{-1} f_{1} h$ can be thought of as an element of $F_{n,\left[-\infty, t_{0}\right]}$. Since $\varphi$ has order $2,\left(f_{2}, f_{1}\right) \in F_{n,\left[-\infty, t_{0}\right]} \times F_{n,\left[t_{0}, \infty\right]}$ is a fixed point of $\varphi$ whose restriction on $J$ is not the identity, giving a contradiction.

The following result yields Proposition B.

Corollary 5.2. The group $C_{F_{n, \infty}}(\varphi)$ is either infinitely generated or of type $\mathrm{F}_{\infty}$

Proof. This follows directly from Corollary 4.15 and the previous lemma. We observe that there is a shorter proof of this corollary avoiding Corollary 4.15. If $C_{F_{n, \infty}}(\varphi)$ is finitely generated the previous lemma implies that there is an element $\hat{f}$ of $C_{F_{n, \infty}}(\varphi)$ which has right-hand slope at $t_{0}$ different from 
1. Note that this is stronger than condition (2), which requires such an element to just lie in $F_{n, \infty}$. This element can be used directly in the proof of Theorem 4.14 to show that the image of the map $\rho$ of the proof of Theorem 4.14 has finite index in $F_{n,\left[t_{0}, \infty\right]}$ instead of using Corollary 4.12. Indeed, define $M$ as the subgroup of $F_{n,\left[t_{0}, \infty\right]}$ containing the elements which have slope 1 at $t_{0}$. Then with the notation used in the proof of Corollary 4.15, $M \cup\{\rho(\hat{f})\} \subseteq \operatorname{Im}(\rho)$ and $\rho(\hat{f}) \notin M$. Thus $M$ is a proper subgroup of $\operatorname{Im}(\rho)$. Hence $1 \neq \operatorname{Im}(\rho) / M \leq F_{n,\left[t_{0}, \infty\right]} / M \simeq \mathbb{Z}$ and $\operatorname{Im}(\rho)$ has finite index in $F_{n,\left[t_{0}, \infty\right]}$. In particular, as $\rho$ is injective, $\operatorname{Im}(\rho) \simeq C_{F_{n, \infty}}(\varphi)$ is of type $\mathrm{F}_{\infty}$ as required.

Corollary 5.3. There exists an element $f \in C_{F_{n, \infty}}(\varphi)$ with right-hand slope at $t_{0}$ different from 1 if and only if there exists an element $f \in F_{n, \infty}$ with right-hand slope at $t_{0}$ different from 1.

Proof. This follows directly from Corollary 4.15 and Lemma 5.1.

\section{Auxiliary Results}

As in [7] we consider the function

$$
\phi_{n}: \mathbb{Z}\left[\frac{1}{n}\right] \rightarrow \mathbb{Z}_{n-1}
$$

given by

$$
\phi_{n}\left(n^{a} b\right) \equiv b \quad(\bmod n-1),
$$

where $a, b \in \mathbb{Z}$ and $b$ is not divisible by $n$.

Lemma 6.1. Let $n, k$ be coprime natural numbers. Consider

$$
t_{1}:=\frac{m}{k} n^{a}, \quad t_{2}:=\frac{r}{k} n^{c}
$$

such that

$$
k(n-1) \mid r n^{c}-m n^{a}
$$

(here, divisibility is understood in $\mathbb{Z}\left[\frac{1}{n}\right]$ ). Then there exists some $g \in F_{n, \infty}$ such that

$$
\left(t_{1}\right) g=t_{2} .
$$

Proof. Let $0<s<k$ such that $1+n s=k t$ for some $t \in \mathbb{Z}$. We assume $t_{1} \geq 0$, the other case is analogous. Put

$$
\begin{gathered}
x_{1}:=t_{1}+t_{1} n s, \\
x_{2}:=t_{1}+t_{1} n(s-k), \\
y_{1}:=t_{2}+t_{1} n s, \\
y_{2}:=t_{2}+t_{1} n(s-k) .
\end{gathered}
$$

Clearly, $x_{2} \leq t_{1} \leq x_{1}$ and $y_{2} \leq t_{2} \leq y_{1}$. Moreover $x_{1}=t_{1} k t \in \mathbb{Z}\left[\frac{1}{n}\right]$ and as $x_{1}-x_{2}=y_{1}-y_{2}=t_{1} n k=m n^{a+1} \in \mathbb{Z}\left[\frac{1}{n}\right]$ and $y_{1}-x_{1}=t_{2}-t_{1}=\frac{r n^{c}-m n^{a}}{k} \in$ $\mathbb{Z}\left[\frac{1}{n}\right]$ we observe that $x_{1}, x_{2}, y_{1}, y_{2} \in \mathbb{Z}\left[\frac{1}{n}\right]$. Also,

$$
\phi_{n}\left(y_{1}-x_{1}\right)=\phi_{n}\left(y_{2}-x_{2}\right)=\phi_{n}\left(\frac{r n^{c}-m n^{a}}{k}\right)=0
$$

by hypothesis which implies $\phi_{n}\left(x_{i}\right)=\phi_{n}\left(y_{i}\right)$ for $i=1,2$. Therefore by [7, Lemma 1.2.1] there is $g \in F_{n, \infty}$ such that $\left(x_{i}\right) g=y_{i}$. Moreover this $g$ in 
the interval $\left[x_{2}, x_{1}\right]$ is constructed as follows: as the length of the interval is $x_{1}-x_{2}=m n^{a+1}$, we may subdivide it in $m$ intervals of length $n^{a+1}$ each. We do the same with $\left[y_{2}, y_{1}\right]$, which has the same length $m n^{a+1}$ and then define $g$ mapping each subdivision of $\left[x_{2}, x_{1}\right]$ to each subdivision of $\left[y_{2}, y_{1}\right]$. Clearly, this implies that on $\left[x_{2}, x_{1}\right], g$ is precisely the function $(x) g=x+t_{2}-t_{1}$. Therefore

$$
\left(t_{1}\right) g=t_{2}
$$

Lemma 6.2. For any $t_{0} \in \mathbb{Q}$ there exist integers $k, s, t>0$ such that $n-1 \mid k$ and

$$
t_{0}=\frac{k}{n^{t}\left(n^{s}-1\right)}
$$

Proof. Put $t_{0}=r / m$ with $n-1 \mid r$ (we don't require $r, m$ coprime) and decompose $m=m_{1} m_{2}$ so that $m_{2}, n$ are coprime and all the primes dividing $m_{1}$ divide also $n$. Then there is some $t>0$ such that $m_{1} \mid n^{t}$ and some $s>0$ such that $n^{s} \equiv 1 \bmod m_{2}$. Therefore $m=m_{1} m_{2} \mid n^{t}\left(n^{s}-1\right)$ and for some $r_{1}$ we have $r_{1} m=n^{t}\left(n^{s}-1\right)$. Putting $k=r_{1} r$ we are done.

Lemma 6.3. Let $t_{0} \in \mathbb{R}$. Then there is some $f \in F_{n, \infty}$ such that $\left(t_{0}\right) f=t_{0}$ and the right-hand slope of $f$ at $t_{0}$ is not one if and only if $t_{0} \in \mathbb{Q}$.

Proof. Assume first that there exists such an element $f$. Then for some interval $I$ with $t_{0} \in I,(x) f=a x+b$ for any $x \in I$ where $a, b \in \mathbb{Q}$ and $a \neq 1$. Then the equation $\left(t_{0}\right) f=t_{0}$ implies $t_{0} \in \mathbb{Q}$.

Next, we assume that $t_{0} \in \mathbb{Q}$ so by Lemma 6.2 we may put $t_{0}=\frac{k}{n^{t}\left(n^{s}-1\right)}$. Let $a=n^{s}=\langle n\rangle, b=-\frac{k}{n^{t}} \in \mathbb{Z}[1 / n]$. Then for any function $f$ such that in a neighbourhood of $t_{0},(x) f=a x+b$ we have $\left(t_{0}\right) f=t_{0}$. We claim that there is some such function in $F_{n, \infty}$. To prove the claim it is sufficient to find $x_{1}, x_{2} \in \mathbb{Z}[1 / n]$ such that $t_{0} \in\left[x_{1}, x_{2}\right]$ and for $y_{i}=a x_{i}+b, i=1,2$

$$
y_{i}-x_{i}=l_{i}(n-1)
$$

for some integers $l_{i}$. Then the function

$$
(x) f=\left\{\begin{array}{l}
x+l_{1}(n-1) \text { if } x \leq x_{1} \\
a x+b \text { if } x_{1}<x \leq x_{2} \\
x+l_{2}(n-1) \text { if } x_{2}<x
\end{array}\right.
$$

is in $F_{n, \infty}$ and satisfies the conditions we wanted. So we need (recall that $n-1 \mid k$ and denote $\left.k_{1}=k /(n-1)\right)$

$$
y_{i}-x_{i}=(a-1) x_{i}+b=\left(n^{s}-1\right) x_{i}-k / n^{t}=l_{i}(n-1) .
$$

Restricting to $x_{i}$ of the shape $\alpha_{i} / n^{t}$ with $\alpha_{i} \in \mathbb{Z}$ this yields

$$
\frac{\left(n^{s-1}+\ldots+n+1\right) \alpha_{i}-k_{1}}{n^{t}}=l_{i} \in \mathbb{Z} .
$$

As $n^{s-1}+\ldots+n+1$ is coprime to $n^{t}$ we may choose infinitely many values of $\alpha_{i}$ having this property. Note also that $\alpha_{1}$ can be chosen arbitrarily small and $\alpha_{2}$ arbitrarily big so that $t_{0} \in\left[x_{1}, x_{2}\right]$. 


\section{Conjugacy Classes}

Definition. Let $\varphi$ be an automorphism of $F_{n, \infty}$ of order 2 given by conjugation with a homeomorphism $h$ of the real line. Let $k>0$ be an integer. We say that $h$ has property (*) for $k$ if for every element $h_{i}$ of order 2 in $F_{n, \infty} \rtimes\langle h\rangle$ the unique $t_{i} \in \mathbb{R}$ such that $\left(t_{i}\right) h_{i}=t_{i}$ belongs to $\frac{1}{k} \mathbb{Z}\left[\frac{1}{n}\right]$.

Note that for $k=k_{1} p$ with $p, n / p \in \mathbb{Z}$, we have

$$
\frac{1}{k} \mathbb{Z}\left[\frac{1}{n}\right] \subseteq \frac{1}{k_{1}} \mathbb{Z}\left[\frac{1}{n}\right]
$$

This implies that if $h$ has property $(*)$ for some $k$, then it also has property (*) for some $k^{\prime}$ which is coprime to $n$.

By Lemma 6.3 condition (2) of Section 4 holds for the order 2 automorphism induced by conjugation with each $h_{i}$ satisfying the above definition.

Lemma 7.1. Let $\varphi$ be an automorphism of $F_{n, \infty}$ of order 2 given by conjugation with a homeomorphism $h$ of the real line having property $(*)$ for some $k$. Let $f, \widetilde{f} \in F_{n, \infty}$ such that $\varphi(f)=f^{-1}, \varphi(\widetilde{f})=\widetilde{f}^{-1}$ and $\left(t_{0}\right) f=\left(t_{0}\right) \widetilde{f}=$ $t_{0}=\left(t_{0}\right) h$. Then there is an element $g \in F_{n, \infty}$ such that $\varphi(g)^{-1} f g=\widetilde{f}$ and $\left(t_{0}\right) g=t_{0}$.

Proof. Note that $f h$ and $\widetilde{f} h$ are elements of order 2 in $F_{n, \infty} \rtimes\langle h\rangle$ and that

$$
\widetilde{\varphi}\left(f_{1}\right)=f_{2}^{-1}
$$

and

$$
\widetilde{\varphi}\left(\widetilde{f}_{1}\right)=\widetilde{f}_{2}^{-1},
$$

where $f=\left(f_{1}, f_{2}\right), \tilde{f}=\left(\widetilde{f}_{1}, \tilde{f}_{2}\right) \in D=F_{n,\left(-\infty, t_{0}\right]} \times F_{n,\left[t_{0}, \infty\right)}$ and $\widetilde{\varphi} \in \operatorname{Aut}(D)$ is given by conjugation with $h$. The automorphism $\widetilde{\varphi}$ is well-defined by Lemma 4.10 as $\left(t_{0}\right) h=t_{0}$.

We shall construct an element $g=\left(g_{1}, g_{2}\right) \in F_{n,\left(-\infty, t_{0}\right]} \times F_{n,\left[t_{0}, \infty\right)}$ with $g \in F_{n, \infty}$ and such that

$$
\widetilde{\varphi}\left(g_{2}\right)^{-1} f_{1} g_{1}=\widetilde{f}_{1}
$$

and

$$
\widetilde{\varphi}\left(g_{1}\right)^{-1} f_{2} g_{2}=\widetilde{f}_{2}
$$

It is sufficient that the first of the above equalities holds. In case $t_{0} \in \mathbb{Z}\left[\frac{1}{n}\right]$, in particular $t_{0}$ can be a break point of $g$, we define $g_{2}=i d$ and $g_{1}=f_{1}^{-1} \widetilde{f}_{1}$. Suppose now that $t_{0} \notin \mathbb{Z}\left[\frac{1}{n}\right]$ and hence $t_{0}$ cannot be a break point of $g$. We can define $g_{1}$ as $f_{1}^{-1} \widetilde{\varphi}\left(g_{2}\right) \widetilde{f}_{1}$ but have to be sure that $g_{2}$ is chosen in such a way that $t_{0}$ is not a break point of $g$ i.e. $\nu_{1}\left(g_{1}\right)=\mu_{1}\left(g_{2}\right)$. Note that by Corollary $4.12, \nu_{1} \widetilde{\varphi}=\widetilde{\varphi}^{*}\left(\nu_{1}\right)=\mu_{1}$. Hence

$$
\begin{aligned}
\nu_{1}\left(g_{1}\right) & =\nu_{1}\left(f_{1}^{-1} \widetilde{\varphi}\left(g_{2}\right) \widetilde{f}_{1}\right) \\
& =\nu_{1}\left(f_{1}^{-1}\right)+\nu_{1}\left(\widetilde{\varphi}\left(g_{2}\right)\right)+\nu_{1}\left(\widetilde{f}_{1}\right) \\
& =-\nu_{1}\left(f_{1}\right)+\nu_{1}\left(\widetilde{f}_{1}\right)+\mu_{1}\left(g_{2}\right) .
\end{aligned}
$$

Furthermore, since $f_{1}=\widetilde{\varphi}\left(f_{2}\right)^{-1}$ we have

$$
\nu_{1}\left(f_{1}\right)=\nu_{1}\left(\widetilde{\varphi}\left(f_{2}\right)^{-1}\right)=-\nu_{1}\left(\widetilde{\varphi}\left(f_{2}\right)\right)=-\mu_{1}\left(f_{2}\right) .
$$


But as $t_{0}$ is not a break point of $f$, we have $\mu_{1}\left(f_{2}\right)=\nu_{1}\left(f_{1}\right)$. Therefore $\nu_{1}\left(f_{1}\right)=0$, and similarly $\nu_{1}\left(\widetilde{f}_{1}\right)=0$. Combining this with the calculations above we obtain

$$
\nu_{1}\left(g_{1}\right)=-\nu_{1}\left(f_{1}\right)+\nu_{1}\left(\widetilde{f}_{1}\right)+\mu_{1}\left(g_{2}\right)=\mu_{1}\left(g_{2}\right) .
$$

Lemma 7.2. Let $h_{1}$ and $h_{2}$ be elements of order 2 in $G=F_{n, \infty} \rtimes\langle h\rangle$, where $h$ has property $(*)$ for some $k$. Let $t_{i}$ be the unique real number such that $\left(t_{i}\right) h_{i}=t_{i}$ for $i=1,2$. If there is some $\hat{g} \in F_{n, \infty}$ such that $\left(t_{1}\right) \hat{g}=t_{2}$, then $h_{1}$ and $h_{2}$ are conjugated in $G$.

Proof. Consider $f \in F_{n, \infty}$ defined by

$$
h_{2}=f h_{1} .
$$

We write $G$ as $F_{n, \infty} \rtimes\left\langle h_{1}\right\rangle$ and $\varphi_{1} \in \operatorname{Aut}\left(F_{n, \infty}\right)$ is conjugation by $h_{1}$. Let $\widetilde{f}=\hat{g} f \varphi_{1}(\hat{g})^{-1}$. Then

$$
\left(t_{1}\right) \tilde{f}=\left(t_{1}\right) \hat{g} f \varphi_{1}(\hat{g})^{-1}=\left(t_{2}\right) f h_{1} \hat{g}^{-1} h_{1}=\left(t_{2}\right) h_{2} \hat{g}^{-1} h_{1}=\left(t_{2}\right) \hat{g}^{-1} h_{1}=t_{1} .
$$

Note that since $h_{2}^{2}=i d$ we have $\varphi_{1}(f)=f^{-1}$, hence $\varphi_{1}(\widetilde{f})=\widetilde{f}^{-1}$. Now by Lemma 7.1 there is a $g \in F_{n, \infty}$ such that $g \widetilde{f} \varphi_{1}(g)^{-1}=i d$. Thus for $\widetilde{g}=g \hat{g}$ we have

$$
\begin{aligned}
i d=g \widetilde{f} \varphi_{1}(g)^{-1}=g \hat{g} f \varphi_{1}(\hat{g})^{-1} \varphi_{1}(g)^{-1} & =\widetilde{g} f \varphi_{1}(\widetilde{g})^{-1}= \\
\widetilde{g} h_{2} h_{1} h_{1}(\widetilde{g})^{-1} h_{1} & =\widetilde{g} h_{2}(\widetilde{g})^{-1} h_{1},
\end{aligned}
$$

hence

$$
h_{1}=h_{1}^{-1}=\widetilde{g}^{-1} h_{2} \widetilde{g}
$$

Theorem 7.3. Let $\varphi$ be an automorphism of $F_{n, \infty}$ of order 2 given by conjugation with a homeomorphism $h$ of the real line. Let $t_{0}$ be the unique real number such that $\left(t_{0}\right) h=t_{0}$.

a) If $h$ is $P L$, then $h$ has property $(*)$ for 2. In particular $t_{0} \in \frac{1}{2} \mathbb{Z}\left[\frac{1}{n}\right]$;

b) If $h$ has property $(*)$ for some integer, then there are only finitely many conjugacy classes of elements of order 2 in $F_{n, \infty} \rtimes\langle h\rangle$.

Proof. a) Let $f \in F_{n, \infty}$ be such that $f h$ has order 2 i.e. $\varphi(f)=f^{-1}$ and $r_{0}$ be the unique real number such that $\left(r_{0}\right) f h=r_{0}$. Suppose that $r_{0} \notin \mathbb{Z}\left[\frac{1}{n}\right]$, otherwise there is nothing to prove. Then for some small $\varepsilon>0$ we have that the restriction of $f$ on $\left[r_{0}-\varepsilon, r_{0}+\varepsilon\right]$ is a linear function, say $n^{s} t+\lambda$ for some $\lambda \in \mathbb{Z}\left[\frac{1}{n}\right], s \in \mathbb{Z}$. Since $h$ is PL, on a neighborhood of $\left(r_{0}\right) h$ we have $(t) h=a_{1} t+b_{1}$, where the slope is in the multiplicative group of $\mathbb{R}$ generated by the prime divisors of $n$ and $\left(\mathbb{Z}\left[\frac{1}{n}\right]\right) h=\mathbb{Z}\left[\frac{1}{n}\right][7$, Lemma 3.2.2].

Since $(x) h f h f=x$ and $\left(r_{0}\right) h^{2} f=\left(r_{0}\right) f=\left(r_{0}\right) h$, we deduce that for $x$ in a small neighbourhood of $\left(r_{0}\right) h$ we have

$$
\begin{gathered}
\left.x=(x) h f h f=n^{s}\left(a_{1}\left(n^{s}\left(a_{1} x+b_{1}\right)+\lambda\right)\right)+b_{1}\right)+\lambda= \\
\left(n^{s} a_{1}\right)^{2} x+\left(n^{s}\right)^{2} a_{1} b_{1}+n^{s} a_{1} \lambda+n^{s} b_{1}+\lambda .
\end{gathered}
$$


Then $\left(n^{s} a_{1}\right)^{2}=1$, hence $n^{s} a_{1}=-1$ since $a_{1}<0$ (remember $h$ is decreasing) i.e. $a_{1}=-n^{-s}$. Since $\left(\mathbb{Z}\left[\frac{1}{n}\right]\right) h=\mathbb{Z}\left[\frac{1}{n}\right]$ we deduce that $b_{1} \in \mathbb{Z}\left[\frac{1}{n}\right]$. Then

$$
r_{0}=\left(r_{0}\right) h^{2}=a_{1}\left(\left(r_{0}\right) h\right)+b_{1}
$$

and

$$
\begin{gathered}
\left(r_{0}\right) h=\left(r_{0}\right) f=n^{s} r_{0}+\lambda=\left(r_{0}\right) h h f=n^{s}\left(a_{1}\left(\left(r_{0}\right) h\right)+b_{1}\right)+\lambda= \\
n^{s} a_{1}\left(\left(r_{0}\right) h\right)+n^{s} b_{1}+\lambda=-\left(r_{0}\right) h+n^{s} b_{1}+\lambda .
\end{gathered}
$$

Thus

$$
\left(r_{0}\right) f=\left(r_{0}\right) h=\frac{1}{2}\left(n^{s} b_{1}+\lambda\right) \in \frac{1}{2} \mathbb{Z}\left[\frac{1}{n}\right] .
$$

Since $r_{0} \notin \mathbb{Z}\left[\frac{1}{n}\right]$ we have $\left(r_{0}\right) f \notin \mathbb{Z}\left[\frac{1}{n}\right]$ and $\left(r_{0}\right) f$ is not a break point of $f^{-1}$. So in a neighborhood of $\left(r_{0}\right) f$ the function $f^{-1}$ is given by $(x) f^{-1}=n^{\alpha} x+\beta$, where $\beta \in \mathbb{Z}\left[\frac{1}{n}\right], \alpha \in \mathbb{Z}$. Thus

$$
r_{0}=\left(r_{0}\right) f f^{-1}=n^{\alpha}\left(\left(r_{0}\right) f\right)+\beta \in \frac{1}{2} \mathbb{Z}\left[\frac{1}{n}\right]
$$

as required. Note that for $n$ even we have $\frac{1}{2} \mathbb{Z}\left[\frac{1}{n}\right] \subseteq \mathbb{Z}\left[\frac{1}{n}\right]$. Thus

$$
t_{0}, r_{0} \in \mathbb{Z}\left[\frac{1}{n}\right] \text { if } n \text { is even. }
$$

b) Let $k$ be an integer such that $h$ has $(*)$ for $k$. By the comment after the definition of propery $(*)$ we may assume that $n, k$ are coprime. Let $h_{1}, h_{2}$ be elements of order 2 in $G=F_{n, \infty} \rtimes\langle h\rangle$. Let $t_{i}$ be the unique real number such that $\left(t_{i}\right) h_{i}=t_{i}$ for $i=1,2$. Put

$$
t_{1}=\frac{m}{k} n^{a}, \quad t_{2}=\frac{r}{k} n^{c}
$$

for certain integers $m, r, a, c$. Then if these integers satisfy

$$
k(n-1) \mid k t_{2}-k t_{1}=r n^{c}-m n^{a}
$$

in $\mathbb{Z}\left[\frac{1}{n}\right]$, by Lemma 6.1 there is some $\hat{g} \in F_{n, \infty}$ such that $\left(t_{1}\right) \hat{g}=t_{2}$. Therefore $h_{1}$ and $h_{2}$ are conjugated by Lemma 7.2. As the number of possible values of $k t_{i}$ in the quotient ring $\mathbb{Z}\left[\frac{1}{n}\right] / k(n-1) \mathbb{Z}\left[\frac{1}{n}\right]$ is finite, the result follows.

\section{Proof of Theorem A}

The first part of Theorem A is Lemma 4.1. The second part of Theorem A follows from Corollary 4.15 and Lemma 5.1.

To show the third part of Theorem A we use that by Theorem 7.3, $t_{0} \in$ $\frac{1}{2} \mathbb{Z}\left[\frac{1}{n}\right]$. Note that if $n$ is even, $t_{0} \in \mathbb{Z}\left[\frac{1}{n}\right]$ and if $n$ is odd we can apply Lemma 6.3. In both cases there is an $f \in F_{n, \infty}$ such that $\left(t_{0}\right) f=t_{0}$ and the righthand slope of $f$ at $t_{0}$ is not 1 . Then part (2) of Theorem A implies that $C_{F_{n, \infty}}(\varphi)$ is of type $\mathrm{F}_{\infty}$. 


\section{Proof of Theorem C and Corollary D}

Theorem C. Any finite extension $G$ of $F_{n, \infty}$ where the elements of finite order act on $F_{n, \infty}$ via conjugation with $P L$ homeomorphisms is of type Bredon $\mathrm{F}_{\infty}$

Proof. By Lemma 4.1 any element of finite order in $\operatorname{Aut}\left(F_{n, \infty}\right)$ has order 2 and is given by conjugation with a decreasing homeomorphism of the real line (of multiplicative order 2). If a product of such two elements has finite order then the product is a conjugation by an increasing function (i.e. a composition of two decreasing function is increasing), so cannot have a finite order unless the product is the trivial element. Thus every finite subgroup $B$ of $G$ has a subgroup $B_{0}$ of index at most two acting trivially on $F_{n, \infty}$.

Let $\pi: G \rightarrow G / F_{n, \infty}$ be the canonical projection and let $P$ and $Q$ be finite subgroups of $G$ such that $\pi(P)=\pi(Q)$. Since $F_{n, \infty}$ does not have non-trivial elements of finite order $F_{n, \infty} \cap P=1=F_{n, \infty} \cap Q$, so we consider $F_{n, \infty} \rtimes P=F_{n, \infty} \rtimes Q$ as a subgroup of $G$. Since there are only finitely many possibilities for $\pi(P)$ it suffices to show that $F_{n, \infty} \rtimes P$ has finitely many conjugacy classes of finite subgroups, so without loss of generality we can assume that $G=F_{n, \infty} \rtimes P$.

Let $P_{0}$ be the subgroup of $P$ of elements acting trivially on $F_{n, \infty}$ via conjugation. Consider the short exact sequence of groups $P_{0} \longmapsto G \rightarrow F_{n, \infty} \rtimes\left(P / P_{0}\right)$. $P / P_{0}$ is either trivial or a cyclic group of order 2 . Hence Theorem $7.3 \mathrm{im}$ plies that there are only finitely many conjugacy classes of finite subgroups in $F_{n, \infty} \rtimes\left(P / P_{0}\right)$. Since $P_{0}$ is finite, this implies that there are only finitely many conjugacy classes of finite subgroups in $G$.

Finally we show that the centraliser $C_{G}(P)$ of $P$ in $G$ is of type $\mathrm{F}_{\infty}$. If $P=P_{0}$ then $C_{G}(P)$ is a finite extension of $F_{n, \infty}$, so is of type $\mathrm{F}_{\infty}$. If $P \neq P_{0}$ let the action of $P / P_{0}$ on $F_{n, \infty}$ be given by conjugation with a real homeomorphism $h$ and define $t_{0}$ as the unique real number such that $\left(t_{0}\right) h=t_{0}$. By Theorem 7.3, $t_{0} \in \frac{1}{2} \mathbb{Z}\left[\frac{1}{n}\right]$ as the hypothesis imply that $h$ is PL. Note that by Lemma 6.3 there is $f \in F_{n, \infty}$ such that $\left(t_{0}\right) f=t_{0}$ and the right-hand slope of $f$ at $t_{0}$ is not 1 . Then for $\varphi \in \operatorname{Aut}\left(F_{n, \infty}\right)$ given by conjugation with $h$ by Theorem A, part(2)

$$
C_{G}(P) \cap F_{n, \infty}=C_{F_{n, \infty}}(P)=C_{F_{n, \infty}}(\varphi)
$$

is of type $\mathrm{F}_{\infty}$. Note that $C_{F_{n, \infty}}(\varphi)$ is a subgroup of finite index in $C_{G}(P)$, hence $C_{G}(P)$ is of type $\mathrm{F}_{\infty}$ as required.

Corollary D. Any finite extension of $F$ is of type Bredon $\mathrm{F}_{\infty}$.

Proof. By [6, Lemma 5.1] for $n=2$ any orientation preserving automorphism $\theta$ is given by conjugation with a PL homeomorphism $g$ of the real line. As an automorphism of finite order is the conjugation with a homeomorphism of the real line $h$ that is the composition of some $g$ as above with the map $h_{0}: x \rightarrow-x$ we deduce that $h$ is PL. Now we can apply the previous theorem.

\section{Exotic Automorphisms}

It was shown in [7, Thm. 6.1.5] that for each $n \geq 3$ there exist "exotic" orientation preserving automorphisms $\theta$ of $F_{n, \infty}$, where exotic means that 
the automorphisms are given by conjugation with a homeomorphism of the real line, which is not PL.

In this section we shall show that for each $n \geq 3$ there exist infinitely many exotic automorphisms $\varphi$ of order 2 in $\operatorname{Aut}\left(F_{n, \infty}\right)$. It will turn out, however, that the unique element fixed by these automorphisms as constructed in Lemma 4.1 is $t_{0}=0$. Then by Theorem A, part $(2) C_{F_{n, \infty}}(\varphi)$ is of type $\mathrm{F}_{\infty}$.

We adopt the notation of [7, Section 2.1, Section 4] and following [7] all homomorphisms, in particular automorphisms, considered in this section act on the right. Consider the PL functions $g_{n, i}$ and $t_{i}$ given by

$$
(x) g_{n, i}=\left\{\begin{array}{l}
x \quad x<i \\
n(x-i)+i \quad i \leq x \leq i+1 \\
x+n-1 \quad x>i+1
\end{array}\right.
$$

and $t_{s}: x \mapsto x+s$ (here $n, s \in \mathbb{Z}$ ). Please note that $t_{i}$ is not to be confused with the element $t_{0} \in \mathbb{R}$ of Lemma 4.1. Then by [7, Lemma 2.1.2]

$$
F_{n, \infty}=\left\langle t_{n-1}, g_{0}, \ldots, g_{n-2}\right\rangle
$$

and $t_{s}^{-1} g_{n, i} t_{s}=g_{n, i+s}$.

Let $A u t^{0}\left(F_{n, \infty}, t_{1}\right)$ denote the set of automorphisms of $F_{n, \infty}$ given by conjugation with a homeomorphism of the real line fixing 0 and commuting with $t_{1}$. For $n \geq 3$ we consider the map constructed in [7, Thm. 4.3.1]

$$
\Lambda_{2, n}: A u t^{0}\left(F_{2, \infty}, t_{1}\right) \rightarrow A u t^{0}\left(F_{n, \infty}, t_{1}\right),
$$

for which the image of every non-trivial element is exotic.

Let $\rho_{j}$ be the automorphism of $F_{j, \infty}$ given by conjugation with $h_{0}: x \mapsto-x$ and choose an arbitrary element $\theta \in A u t^{0}\left(F_{2, \infty}, t_{1}\right)$ such that $\left(\theta \rho_{2}\right)^{2}=i d$ (for the existence of a suitable $\theta$, see the end of page 17). Denote by $\theta_{n}=$ $\Lambda_{2, n}(\theta)$. We now show that $\left(\theta_{n} \rho_{n}\right)^{2}=i d$, hence $\theta_{n} \rho_{n} \in A u t^{0}\left(F_{n, \infty}, t_{1}\right)$ is an exotic element of finite order.

A simple calculation shows that for $n \geq 2$

$$
\left(g_{n, i}\right) \rho_{n}=g_{n,-i-1} t_{n-1}^{-1} .
$$

Write $\left(g_{2,0}\right) \theta=w\left(g_{2,0}, t_{1}\right)$ as a word in the generators $g_{2,0}$ and $t_{1}$. For all $0 \leq i<n$ the proof of $[7,4.3 .1]$ together with $[7,4.1 .2]$ imply that

$$
\left(g_{n, i}\right) \theta_{n}=w\left(g_{n, i}, t_{n-1}\right) .
$$

Note also that for $n \geq 2$

$$
\left(t_{i}\right) \rho_{n}=t_{i}^{-1}
$$

and since $t_{n-1} \in\left\langle t_{1}\right\rangle$, for $n \geq 3$ we have

$$
\left(t_{n-1}\right) \theta_{n}=t_{n-1} \text {. }
$$

Furthemore

$$
\left(g_{2,0}\right) \theta \rho_{2}=\left(w\left(g_{2,0}, t_{1}\right)\right) \rho_{2}=w\left(\left(g_{2,0}\right) \rho_{2},\left(t_{1}\right) \rho_{2}\right)=w\left(g_{2,-1} t_{1}^{-1}, t_{1}^{-1}\right),
$$

and similarly for every $i \geq 0$ and $n \geq 3$

$$
g_{n, i}\left(\theta_{n} \rho_{n}\right)=w\left(g_{n, i}, t_{n-1}\right) \rho_{n}=w\left(g_{n, i} \rho_{n}, t_{n-1} \rho_{n}\right)=w\left(g_{n,-i-1} t_{n-1}^{-1}, t_{n-1}^{-1}\right) .
$$

Claim. $g_{n, 0}\left(\theta_{n} \rho_{n}\right)^{2}=g_{n, 0}$. 
We observe that

$$
\begin{aligned}
g_{n, 0}\left(\theta_{n} \rho_{n}\right)^{2} & =w\left(g_{n,-1} t_{n-1}^{-1}, t_{n-1}^{-1}\right) \theta_{n} \rho_{n} \\
& =w\left(t_{n-1} g_{n, n-2} t_{n-1}^{-2}, t_{n-1}^{-1}\right) \theta_{n} \rho_{n} \\
& =w\left(\left(t_{n-1} g_{n, n-2} t_{n-1}^{-2}\right) \theta_{n} \rho_{n},\left(t_{n-1}^{-1}\right) \theta_{n} \rho_{n}\right) \\
& =w\left(t_{n-1}^{-1}\left(g_{n, n-2}\right)\left(\theta_{n} \rho_{n}\right) t_{n-1}^{2}, t_{n-1}\right) \\
& =w\left(t_{n-1}^{-1} w\left(g_{n,-(n-1)} t_{n-1}^{-1}, t_{n-1}^{-1}\right) t_{n-1}^{2}, t_{n-1}\right) \\
& =w\left(t_{n-1}^{-1} w\left(t_{n-1} g_{n, 0} t_{n-1}^{-2}, t_{n-1}^{-1}\right) t_{n-1}^{2}, t_{n-1}\right) .
\end{aligned}
$$

Similarly using that $\left(\theta \rho_{2}\right)^{2}=i d$ we have

$$
\begin{aligned}
g_{2,0} & =g_{2,0}\left(\theta \rho_{2}\right)^{2} \\
& =w\left(g_{2,-1} t_{1}^{-1}, t_{1}^{-1}\right) \theta \rho_{2} \\
& =w\left(t_{1} g_{2,0} t_{1}^{-2}, t_{1}^{-1}\right) \theta \rho_{2} \\
& =w\left(\left(t_{1} g_{2,0} t_{1}^{-2}\right) \theta \rho_{2},\left(t_{1}^{-1}\right) \theta \rho_{2}\right) \\
& =w\left(t_{1}^{-1}\left(g_{2,0}\right)\left(\theta \rho_{2}\right) t_{1}^{2}, t_{1}\right) \\
& =w\left(t_{1}^{-1} w\left(g_{2,-1} t_{1}^{-1}, t_{1}^{-1}\right) t_{1}^{2}, t_{1}\right) \\
& =w\left(t_{1}^{-1} w\left(t_{1} g_{2,0} t_{1}^{-2}, t_{1}^{-1}\right) t_{1}^{2}, t_{1}\right) .
\end{aligned}
$$

Now consider the subgroup $H$ of $F_{n, \infty}$ generated by $g_{n, 0}$ and $t_{n-1}$. There is an isomorphism $\nu: H \rightarrow F_{2, \infty}$ given by $g_{n, 0} \nu=g_{2,0}$ and $t_{n-1} \nu=t_{1}$. Applying (4) and (5) yields

$$
\begin{aligned}
\left(g_{n, 0}\left(\theta_{n} \rho_{n}\right)^{2}\right) \nu & =\left(w\left(t_{n-1}^{-1} w\left(t_{n-1} g_{n, 0} t_{n-1}^{-2}, t_{n-1}^{-1}\right) t_{n-1}^{2}, t_{n-1}\right)\right) \nu \\
& =w\left(t_{1}^{-1} w\left(t_{1} g_{2,0} t_{1}^{-2}, t_{1}^{-1}\right) t_{1}^{2}, t_{1}\right) \\
& =g_{2,0}=g_{n, 0} \nu .
\end{aligned}
$$

Hence the claim

$$
g_{n, 0}\left(\theta_{n} \rho_{n}\right)^{2}=g_{n, 0} .
$$

follows. Finally, for all $i>0$ we obtain

$$
g_{n, i}\left(\theta_{n} \rho_{n}\right)^{2}=\left(g_{n, 0}^{t_{1}^{i}}\right)\left(\theta_{n} \rho_{n}\right)^{2}=g_{n, 0}^{t_{1}^{i}\left(\theta_{n} \rho_{n}\right)^{2}}=g_{n, 0}^{t_{1}^{i}}=g_{n, i},
$$

which yields

$$
\left(\theta_{n} \rho_{n}\right)^{2}=i d
$$

as required.

Recall, that by definition $\theta$ is given by conjugation with an orientation preserving homeomorphism $f_{\theta}$ of the real line fixing 0 and commuting with $t_{1}$. So we have for any $s \in \mathbb{Z}$

$$
(x+s) f_{\theta}=(x) f_{\theta}+s .
$$

The condition $\left(\theta \rho_{2}\right)^{2}=i d$ is equivalent with $\left(f_{\theta} h_{0}\right)^{2}=1$ and (6) implies that this is equivalent with requiring that for all $x \in[0,1]$

$$
\left(1-(1-x) f_{\theta}\right) f_{\theta}=x .
$$

By [6, Lemma 5.1] $f_{\theta}$ is PL and furthermore, by $[6$, Thm. 1, iv)] its restriction $f_{1}$ to $[0,1]$ is in $P L_{2}[0,1]$. Hence $f_{1}$ is an element of $F=F_{2, \infty}$ via its realization on the unit interval. 
There are infinitely many elements $f_{1} \in F$ that via the outer automorphism given by conjugation with $x \rightarrow 1-x$ (i.e. flipping over the interval) are sent to $f_{1}^{-1}$. Any such $f_{1}$ gives rise to some $\theta$ whose image $\Lambda_{2, n}(\theta)$ is not PL and such that $\left(\Lambda_{2, n}(\theta) \rho_{n}\right)^{2}=i d$. Hence we have produced infinitely many automorphisms of order 2 of $\operatorname{Aut}\left(F_{n, \infty}\right)$, which are not PL. But, as mentioned above, this way we are not going to obtain potential examples $\varphi$ of finite order in $\operatorname{Aut}\left(F_{n, \infty}\right)$, for which $C_{F_{n, \infty}}(\varphi)$ is not finitely generated.

\section{REFERENCES}

[1] M. Bestvina, N. Brady, Morse theory and finiteness properties of groups, Invent. Math. 129 (1997), no. 3, 445-470

[2] R. Bieri, R. Geoghegan, Sigma invariants of direct products of groups, to appear in Groups, Geometry, Dynamics

[3] R. Bieri, R. Geoghegan, D. Kochloukova, The Sigma invariants of Thompson group $F$, to appear in Groups, Geometry, Dynamics

[4] R. Bieri, W. D. Neumann, R. Strebel A geometric invariant of discrete groups, Invent. Math. 90 (1987), no. 3, 451-477

[5] R. Bieri, B. Renz Valuations on free resolutions and higher geometric invariants of groups, Comment. Math. Helv. 63 (1988), no. 3, 464-497

[6] M. G. BrinThe chameleon groups of Richard J. Thompson: automorphisms and dynamics., Inst. Hautes tudes Sci. Publ. Math. No. 84 (1996), 5-33

[7] M. G. Brin, F. Guzmán Automorphisms of generalised Thompson groups, J. Algebra 203 (1998), no. 1, 285-348

[8] K. S. Brown, R. Geoghegan, An infinite-dimensional torsion-free $\mathrm{FP}_{\infty}$ group, Invent. Math. 77 (1984), no. 2, 367-381

[9] K. S. Brown Finiteness properties of groups, Proceedings of the Northwestern conference on cohomology of groups (Evanston, Ill., 1985), J. Pure Appl. Algebra 44 (1987), no. 1-3, 45-75

[10] J. W. Cannon, W. J. Floyd, W. R. Parry, Introductory notes on Richard Thompson's groups, Enseign. Math. (2) 42 (1996), no. 3-4, 215-256

[11] P. H. Kropholler, C. Martínez-Pérez, B. E. A. Nucinkis, Cohomological finiteness conditions for elementary amenable groups, J. reine angew. Math. 637 (2009), 49-62.

[12] I. J. Leary, B. E. A. Nucinkis, Some groups of type VF, Invent. Math. 151 (2003), no. $1,135-165$

[13] W. Lück, The type of the classifying space for a family of subgroups, J. Pure Appl. Algebra 149 (2000), no. 2, 177-203

[14] H. Meinert, The homological invariants for metabelian groups of finite Prüfer rank: a proof of the $\Sigma^{m}$-conjecture. Proc. London Math. Soc. (3) 72 (1996), no. 2, 385-424

[15] H. Meinert, Actions on 2-complexes and the homotopical invariant $\Sigma^{2}$ of a group. J. Pure Appl. Algebra 119 (1997), no. 3, 297-317

[16] C. Martínez-Pérez, B. E. A. Nucinkis , Virtually soluble groups of type $\mathrm{FP}_{\infty}$, Comment. Math. Helv. 85 (2010), no. 1, 135-150.

[17] S. H. McCleary, M. Rubin, Locally moving groups and the reconstruction problem for chains and circles, preprint, Bowling Green State University, Bowling Green, Ohio

[18] D. Meintrup and T. Schick, A model for the universal space for proper actions of a hyperbolic group. New York J. Math., 8:17 (electronic), 2002.

[19] G. Mislin, Equivariant K-homology of the classifying space for proper actions, Notes on an Advanced Course on Proper Group Actions, CRM Barcelona (2001) 7-86.

[20] R. Gehrke, The higher geometric invariants for groups with sufficient commutativity, Comm. Algebra 26 (1998), no. 4, 1097-1115

[21] M. Stein, Groups of piecewise linear homeomorphisms, Trans. Amer. Math. Soc. 332 (1992), no. 2, 477-514 
Dessislava H. Kochloukova, Department of Mathematics, University of CampINAS, CX. P. 6065, 13083-970 CAMPINAS, SP, BRAZIL

E-mail address: desi@unicamp.br

Conchita Martínez-PÉrez, Departamento de Matemáticas, Universidad de Zaragoza, 50009 Zaragoza, Spain

E-mail address: conmar@unizar.es

Brita E. A. Nucinkis, School of Mathematics, University of Southampton, Southampton, SO17 1BJ, United Kingdom

E-mail address: bean@soton.ac.uk 